\title{
A 72-Year-Old Patient with Longstanding, Untreated Familial Hypercholesterolemia but no Coronary Artery Calcification: A Case Report
}

\author{
Kipp W. Johnson ${ }^{1}$, Joel T. Dudley ${ }^{2}$, Jason R. Bobe ${ }^{3}$ \\ 1. Institute for Next Generation Healthcare, Icahn School of Medicine at Mount Sinai, New York, N.Y., \\ USA, New York, USA 2. Institute for Next Generation Healthcare, Icahn School of Medicine at Mount \\ Sinai, New York, USA 3. Icahn Institute, Genetic and Genomic Sciences, Icahn School of Medicine at \\ Mount Sinai, New York, USA
}

$\square$ Corresponding author: Jason R. Bobe, jason.bobe@mssm.edu

Disclosures can be found in Additional Information at the end of the article

\section{Abstract}

Familial hypercholesterolemia (FH) is a genetic disease associated with persistently elevated levels of low-density lipoprotein cholesterol (LDL-C), which ultimately leads to greatly increased rates of atherosclerosis and cardiovascular disease. Atherosclerosis progression can be clinically approximated through measurement of coronary artery calcification (CAC). CAC can be measured via electron beam computed tomography (EBCT), multi-slice computed tomography (MSCT), or contrast-enhanced CT coronary angiography (CTCA). Here, we present the case of a 72-year-old man with known FH and established hypercholesterolemia who has consistently tested negative for any significant CAC.

Received 03/29/2018

Review began 04/02/2018 Review ended 04/06/2018 Published 04/09/2018

(C) Copyright 2018

Johnson et al. This is an open access article distributed under the terms of the Creative Commons Attribution License CC-BY 3.0., which permits unrestricted use, distribution, and reproduction in any medium, provided the original author and source are credited.
Categories: Cardiology, Genetics, Internal Medicine

Keywords: incomplete penetrance, coronary artery calcification, familial hypercholesterolemia, cardiovascular disease, ldlr mutation, genetic resilience, high cholesterol, positive outlier, resilience

\section{Introduction}

Classical familial hypercholesterolemia (FH) is an autosomal semidominant genetic disease characterized primarily by persistent elevations ( $>310 \mathrm{mg} / \mathrm{dL}$ ) in low-density lipoprotein cholesterol (LDL-C) [1]. FH is most often caused by mutations in the gene encoding the LDL receptor $(L D L R)$ and phenotypic penetrance for damaging variants is nearly complete, with approximately $90 \%$ of heterozygotes eventually developing $\mathrm{FH}$ [2]. Elevated levels of LDL-C have been shown via epidemiological and genetic studies to cause atherosclerosis and coronary artery disease (CAD). Consequently, both treated and untreated familial hypercholesterolemia are associated with greatly elevated risk for CAD, with estimated odds ratios of 10.3 (95 CI: 7.813.8) and 13.2 (10.0-17.4) respectively compared to non-FH patients [3]. Most patients with monogenic FH are heterozygous, and individuals with homozygous mutations display an even more severe damaging phenotype [4]. More recently, it has been suggested that a significant fraction of individuals with $\mathrm{FH}$ and no apparent monogenic mutation may have a polygenic etiology resulting from an excess of mildly deleterious variants in a number of genes, although the true proportion of FH with this polygenic etiology is still an area of active research.

Coronary artery calcification (CAC) is an early sign of the presence of subclinical atherosclerotic disease. CAC, typically assessed via cardiac computed tomography (CT) or electron beam CT, is 
often observed early in patients with FH due to increased LDL-C burden [5]. Here, we present the case of a 72-year-old patient with untreated FH but no evidence of CAD on six separate CAC tests between late 1990s and 2018.

\section{Case Presentation}

Our patient is a normotensive (clinic blood pressure: 110/70) 72-year-old man with a body mass index (BMI) of 25.0 who is heterozygous for a known pathogenic mutation in $L D L R$ (rs879254637) originally reported in 2007 [6]. This mutation consists of a 3-basepair deletion and 14-basepair insertion at position 11,105,586 (GRCh38.p7 reference genome) resulting in a frameshift mutation with a new stop codon at position 42 and ultimately a nonfunctional $L D L R$ protein product. This is a rare variant, as there are no other examples of this variant in the gnomAD genome database [7].

The subject has a longstanding history of hypercholesterolemia. He was initially diagnosed while in his first or second year as a college student after presenting with corneal arcus and LDL-C levels above $300 \mathrm{mg} / \mathrm{dL}$. His initial therapy consisted of clofibrate. He later switched to six packets per day of cholestyramine and niacin (nicotinic acid) which he tolerated poorly and which were ultimately discontinued when he switched to statins. In the 1980s, he participated in early trials for statins (3-hydroxy-3-methylglutaryl-CoA (HMG-CoA) reductase inhibitors) in the United States and from the 1980s through 2006 he was treated with a statin therapy of progressively increasing dosage and intensity. When atorvastatin became commercially available he switched to it at maximum dosage (80 mg), in combination with niacin for a short while. He reports that pharmacologic therapy with statins was largely ineffective at reducing his LDL-C levels, with the majority of lab results reporting results above $300 \mathrm{mg} / \mathrm{dL}$ and a single lowest value of $260 \mathrm{mg} / \mathrm{dL}$ while on combination atorvastatin and niacin. In addition to FH-directed therapy, our subject reports occasionally using baby aspirin (81 mg) and over-thecounter Vitamin D supplements and multivitamins.

In the early 1990s, our patient underwent electron beam computed tomography (EBCT) imaging for CAC following a series of elevated lipid panels. Presence of CAC was assessed in the left main, left anterior descending, left circumflex, and right coronary arteries and scored using the Agatston score. His initial score was 0.0, implying a greater than 95\% chance of absence of coronary artery disease. Because of this surprising finding, he subsequently undertook four additional EBCT tests from 2006 to 2014 resulting in Agatston scores of 1.6, 2.1, 0.0, and 0.0, suggesting a nearly complete absence of any coronary artery calcification. In February of 2018, he underwent multi-slice CT which revealed a complete absence of coronary artery calcification, although mild aortic calcification was observed (Figure 1). His Agatston score was again 0 . 


\section{Cureus}

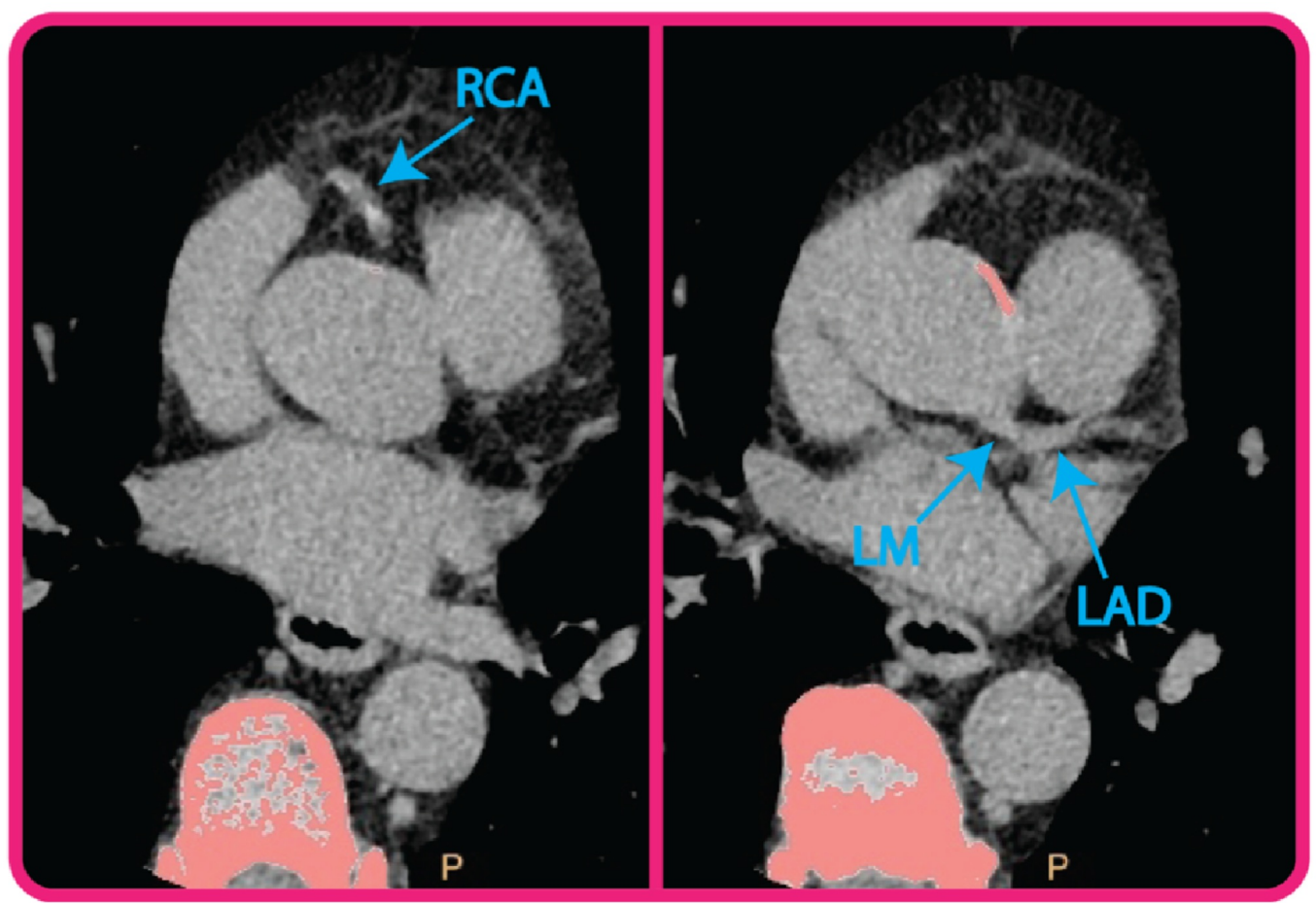

\section{FIGURE 1: Cardiac computed tomography (CT) results.}

Pictured are two CT transverse slices from 2018 (patient age: 72) demonstrating the absence of calcification in the right coronary artery (RCA) and left main (LM) and left anterior descending (LAD) coronary arteries.

We obtained detailed lipid testing results taken between 2009 and 2018 (Table 1). In his panels between 2009 and 2018, LDL-C concentrations were measured at $486.9 \pm 30 \mathrm{mg} / \mathrm{dL}$. Our patient also underwent advanced lipid panel testing (which revealed elevated levels of apolipoprotein $\mathrm{B}$ (ApoB, $297.8 \pm 17.6 \mathrm{mg} / \mathrm{dL}$ ), mildly elevated levels of lipoprotein-associated phospholipase A2 (Lp-PLA2, $212.8 \pm 4.6 \mathrm{ng} / \mathrm{mL})$, and mildly elevated triglycerides $(134.6 \pm 38.9 \mathrm{mg} / \mathrm{dL})$. The patient's mean high-density lipoprotein (HDL) was $67.5 \pm 7.2 \mathrm{mg} / \mathrm{dL}$. Mean lipoprotein(a) (Lp(a)) levels were $4 \pm 1.4 \mathrm{mg} / \mathrm{dL}$. LDL particle subtyping revealed normal values for atherogenic LDL particles IIIa and IVb, and the majority of LDL was of the large Type I LDL. Complete blood count and comprehensive metabolic panel were generally normal except for mildly elevated blood urea nitrogen (25 mg/dL). Genotyping at ApoE (3/3) and KIF6 (Trp/Trp) revealed the absence of risk alleles. No pathogenic variants were identified in the genes PCSK9, APOB, or LDLRAP1.

\section{(A) Longitudinal Laboratory Values}

$\begin{array}{lcccccccc}\text { Date } & 2009 & 2010 & 2011 & 2012 & 2013 & 2014 & 2018 & \text { Mean (SD) } \\ \text { Total Cholesterol (mg/dL) } & 542 & 578 & 562,636 & 599 & 617 & 561 & 564 & 582.4(31.2) \\ \text { LDL (mg/dL) } & 450 & 484 & 470,535 & 506 & 519 & 461 & 470 & 486.9(30.0) \\ \text { HDL (mg/dL) } & 60 & 69 & 73,75 & 76 & 68 & 62 & 57 & 67.5(7.2) \\ \text { Triglycerides (mg/dL) } & 113 & 124 & 96,130 & 86 & 150 & 191 & 187 & 132.6(38.9) \\ \text { Lp(a) (mg/dL) } & 6 & 3 & - & 3 & 4 & - & - & 4(1.4)\end{array}$




\section{Cureus}

\begin{tabular}{|c|c|c|c|c|c|c|c|c|}
\hline Lp-PLA2 (ng/mL) & - & 213 & 208 & 211 & 219 & - & - & $212.8(4.6)$ \\
\hline ApoB (mg/dL) & - & 288 & 324 & 288 & 291 & - & - & $297.8(17.6)$ \\
\hline Insulin ( $\mu \mathrm{IU} / \mathrm{mL})$ & - & 8 & 5 & 5 & 8 & - & 17 & $8.6(4.9)$ \\
\hline LDL Pattern & A & A & A & A & A & - & - & - \\
\hline LDL Particle No. & - & - & - & - & - & $>3500$ & $>3500$ & - \\
\hline Small LDL Particle No. & - & - & - & - & - & 2300 & 2215 & $2257.5(60.1)$ \\
\hline LDL Particle Size $(\AA)$ & - & 273 & 278 & 281 & 276 & 268 & 214 & 265 (25.4) \\
\hline HDL Particles, Total $(\mu \mathrm{mol} / \mathrm{L})$ & - & - & - & - & - & 26.8 & 25.3 & $26.1(1.1)$ \\
\hline \multicolumn{9}{|l|}{ (B) Advanced Lipid Testing } \\
\hline Date & 2009 & 2010 & 2011 & 2012 & 2013 & 2014 & 2018 & Mean (SD) \\
\hline LDL-1 (\%) & - & 1.6 & 3.9 & 3.9 & 2.3 & - & - & $2.9(1.2)$ \\
\hline LDL-Ila (\%) & - & 0.1 & 0.5 & 0.3 & 0.4 & - & - & $0.3(0.2)$ \\
\hline LDL-IIb (\%) & - & 4.8 & 8.8 & 7.2 & 6.7 & - & - & $6.9(1.6)$ \\
\hline LDL-IIIa (\%) & - & 1.2 & 3.0 & 3.1 & 1.8 & - & - & $2.3(0.9)$ \\
\hline LDL-IIIb (\%) & - & 0.4 & 0.9 & 0.8 & 0.5 & - & - & $0.6(0.2)$ \\
\hline LDL IIIa+IIIb (\%) & - & 1.6 & 3.9 & 3.9 & 2.3 & - & - & $2.9(1.2)$ \\
\hline LDL-IVa (\%) & - & 0.2 & 0.8 & 0.7 & 0.5 & - & - & $0.6(0.3)$ \\
\hline LDL-IVb (\%) & - & 0.1 & 0.5 & 0.3 & 0.4 & - & - & $0.3(0.2)$ \\
\hline Q-LDL IIla+|llb (mg/dL) & - & 5.6 & 15.0 & 13.4 & 8.0 & - & - & $10.5(4.4)$ \\
\hline Q-LDL IVb (mg/dL) & - & 0.1 & 2.3 & 1.3 & 1.7 & - & - & $1.3(0.9)$ \\
\hline HDL-2a (\%) & - & 23 & 26 & 21 & 24 & - & - & $23.5(2.1)$ \\
\hline HDL-2b (\%) & - & 23 & 23 & 24 & 23 & - & - & $23.3(0.5)$ \\
\hline HDL-3a (\%) & - & 31 & 27 & 27 & 28 & - & - & $28.2(1.9)$ \\
\hline HDL-3b (\%) & - & 19 & 16 & 17 & 18 & - & - & $17.5(1.3)$ \\
\hline HDL-3c (\%) & - & 3 & 8 & 10 & 8 & - & - & $7.2(3.0)$ \\
\hline
\end{tabular}

\section{TABLE 1: Documented lab values with corresponding dates.}

LDL: Low-density lipoprotein; HDL: High-density lipoprotein; LP(a): Lipoprotein (a); LP-PLA2: Lipoprotein-associated phospholipase A2; ApoB: Apolipoprotein B; Q-LDL: Quantitative LDL. 
diet. Before this, he reports that he generally ate a low-fat, high-carbohydrate diet. He reports drinking on average three glasses of wine per week. He has never smoked and drinks five to six cups of coffee per day on average. Past medical history includes obstructive sleep apnea managed with a continuous positive airway pressure (CPAP) machine, appendicitis at age 18, diverticulitis, a ruptured Achilles tendon attributed to diverticulitis treatment with ciprofloxacin, and intestinal adhesions and surgically repaired intestinal volvulus. The patient also reports that he had a prostatectomy in 2016 related to cancer. He consistently has had blood glucose levels between 100 and $105 \mathrm{mg} / \mathrm{dL}$ for the past decade. The patient denies any significant experiences of chest pain or other symptoms implying angina or ischemia, although he has had a few isolated episodes of palpitations. He has one sister three years his senior who also has FH and a history of high lipid levels. She also has no history of myocardial infarction, angina, or other symptoms of coronary artery disease. His mother had FH, although she died of pancreatic cancer at age 77 . She and her three siblings were never treated for, and had no history of, cardiovascular disease. The patient reports that his father had one high cholesterol score (290s), but was never diagnosed with FH, had no history of cardiovascular disease and died in his 80s during surgery for hernia repair. Our patient has two children, neither of whom have FH.

\section{Discussion}

Our patient is a 72-year-old man affected with a known heterozygous, damaging $L D L R$ mutation causing familial hypercholesterolemia and the resulting expected elevated levels of serum cholesterol. Despite his lipid history, our patient has evidence for the absence of any significant atherosclerotic disease as assessed via repeated EBCT coronary artery calcification imaging over several years with Agatston scores of $0,1,2,0$, and 0 . In comparison, it has been estimated that the median Agatston score for white males between the age of 65 and 74 is 145 [8]. In fact, our patient's mean Agatston score of $<1$ is below the median for even low risk, healthy controls in the closest comparable age reference bracket for which there were large enough numbers (55-64 years old; median score 13) [8].

This is a surprising result. Patients with damaging FH-related mutations and longstanding elevations in lipid levels are expected to experience both premature CAC as well as a greater extent of CAC compared to age-matched controls [5]. CAC risk is even higher in patients with monogenic FH, as our patient possesses, when compared to patients with polygenic CAC [9]. Additionally, it has been noted that patients with a long history of high-intensity statin therapy, as with our patient, are expected to have higher levels of CAC as assessed by EBCT [10]. Our patient's mildly elevated levels of Lp-PLA2 and ApoB are also associated with elevated $\mathrm{CAC}$, although evidence for these newer biomarkers is still emerging.

Essentially, our patient demonstrates an absence of atherosclerotic disease compared to even healthy individuals despite a longstanding history of familial hypercholesterolemia and the presence of a number of cardiovascular risk factors. However, we also note that our patient demonstrates a number of protective factors and behaviors. For example, our subject has a normal BMI; is not hypertensive; has never smoked cigarettes; eats a high-fat, lowcarbohydrate diet; and also possesses a strongly positive life outlook. Taken altogether, we hypothesize that a multitude of protective factors (or on the contrary, the absence of damaging risk factors) could contribute to what we have christened a "polyprotective" phenotype. Furthermore, the absence of significant cardiovascular events in his family members who also have FH implies that there could be a genetic basis for his evasion of adverse cardiovascular outcomes.

We are currently in the process of designing further assays to attempt to identify the factors protecting him from coronary artery calcification. Hypotheses involve each step in the biomolecular pathogenic process for coronary artery disease: LDL particle morphology and 
function, LDL oxidation and macrophage function, chronic inflammation, and vascular hemodynamics. Most tantalizingly, we intend to perform whole-genome sequencing on our subject with the hope of identifying novel mutations which could be counteracting his damaging loss-of-function mutation in $L D L R$. This would be analogous to the early $2000 \mathrm{~s}$ discovery of Sharlayne Tracy's mutation in the protein Proprotein convertase subtilisin/kexin type 9 (PCSK9), which led to the successful clinical development of the anti-lipid PCSK9inhibiting drugs alirocumab and evolocumab.

\section{Conclusions}

We have identified a man with a longstanding history of elevated lipid levels caused by a damaging variant in the low-density lipoprotein receptor gene. Despite his condition, our patient has a surprising complete absence of significant coronary artery calcification. Further efforts are underway to interrogate why our patient has escaped the damaging consequences of familial hypercholesterolemia and could inform future efforts in drug discovery and therapy development.

\section{Additional Information}

\section{Disclosures}

Human subjects: Consent was obtained by all participants in this study. Program for the Protection of Human Subjects/Institutional Review Board, Icahn School of Medicine at Mount Sinai issued approval 15-1503. This study has been approved by the Program for the Protection of Human Subjects/Institutional Review Board at the Icahn School of Medicine at Mount Sinai, New York, NY, USA. Conflicts of interest: In compliance with the ICMJE uniform disclosure form, all authors declare the following: Payment/services info: All authors have declared that no financial support was received from any organization for the submitted work. Financial relationships: All authors have declared that they have no financial relationships at present or within the previous three years with any organizations that might have an interest in the submitted work. Other relationships: All authors have declared that there are no other relationships or activities that could appear to have influenced the submitted work.

\section{Acknowledgements}

We would like to thank the patient for his exceptional assistance and for providing ongoing feedback. We would also like to thank the Resilience Project Study team, especially Eddye Golden for her assistance in record collection.

\section{References}

1. Nordestgaard BG, Chapman MJ, Humphries SE, et al.: Familial hypercholesterolaemia is underdiagnosed and undertreated in the general population: guidance for clinicians to prevent coronary heart disease: consensus statement of the European Atherosclerosis Society. Eur Heart J. 2013, 34:3478-3490. 10.1093/eurheartj/eht273

2. Do R, Willer CJ, Schmidt EM, et al.: Common variants associated with plasma triglycerides and risk for coronary artery disease. Nat Genet. 2013, 45:1345-1352. 10.1038/ng.2795

3. Wong B, Kruse G, Kutikova L, Ray KK, Mata P, Bruckert E: Cardiovascular disease risk associated with familial hypercholesterolemia: a systematic review of the literature. Clin Ther. 2016, 38:1696-1709. 10.1016/j.clinthera.2016.05.006

4. Thompson GR, Blom DJ, Marais AD, Seed M, Pilcher GJ, Raal FJ: Survival in homozygous familial hypercholesterolaemia is determined by the on-treatment level of serum cholesterol. Eur Heart J. 2017, 39:1162-1168. 10.1093/eurheartj/ehx317

5. Gallo A, Giral P, Carrie A, et al.: Early coronary calcifications are related to cholesterol burden in heterozygous familial hypercholesterolemia. J Clin Lipidol. 2017, 11:704-711.

10.1016/j.jacl.2017.03.016 


\section{Cureus}

6. Taylor A, Tabrah S, Wang D, et al.: Multiplex ARMS analysis to detect 13 common mutations in familial hypercholesterolaemia. Clin Genet. 2007, 71:561-568. 10.1111/j.1399-

0004.2007.00807.x

7. Lek M, Karczewski KJ, Minikel EV, et al.: Analysis of protein-coding genetic variation in 60,706 humans. Nature. 2016, 536:285-291. 10.1038/nature19057

8. McClelland RL, Chung H, Detrano R, Post W, Kronmal RA: Distribution of coronary artery calcium by race, gender, and age: results from the Multi-Ethnic Study of Atherosclerosis (MESA). Circulation. 2006, 113:30-37. 10.1161/CIRCULATIONAHA.105.580696

9. Sharifi M, Higginson E, Bos S, et al.: Greater preclinical atherosclerosis in treated monogenic familial hypercholesterolemia vs. polygenic hypercholesterolemia. Atherosclerosis. 2017, 263:405-411. 10.1016/j.atherosclerosis.2017.05.015

10. Henein M, Granasen G, Wiklund U, Schmermund A, Guerci A, Erbel R, Raggi P: High dose and long-term statin therapy accelerate coronary artery calcification. Int J Cardiol. 2015, 184:581586. 10.1016/j.ijcard.2015.02.072 